This item was submitted to Loughborough's Research Repository by the author.

Items in Figshare are protected by copyright, with all rights reserved, unless otherwise indicated.

\title{
Condition monitoring of rail vehicle bogies
}

PLEASE CITE THE PUBLISHED VERSION

http://dx.doi.org/10.1049/ic.2010.0447

PUBLISHER

(c) IEEE

VERSION

AM (Accepted Manuscript)

LICENCE

CC BY-NC-ND 4.0

REPOSITORY RECORD

Ward, Christopher P., Roger M. Goodall, Roger Dixon, and Guy Charles. 2019. "Condition Monitoring of Rail Vehicle Bogies". figshare. https://hdl.handle.net/2134/6640. 
This item was submitted to Loughborough's Institutional Repository (https://dspace.lboro.ac.uk/) by the author and is made available under the following Creative Commons Licence conditions.

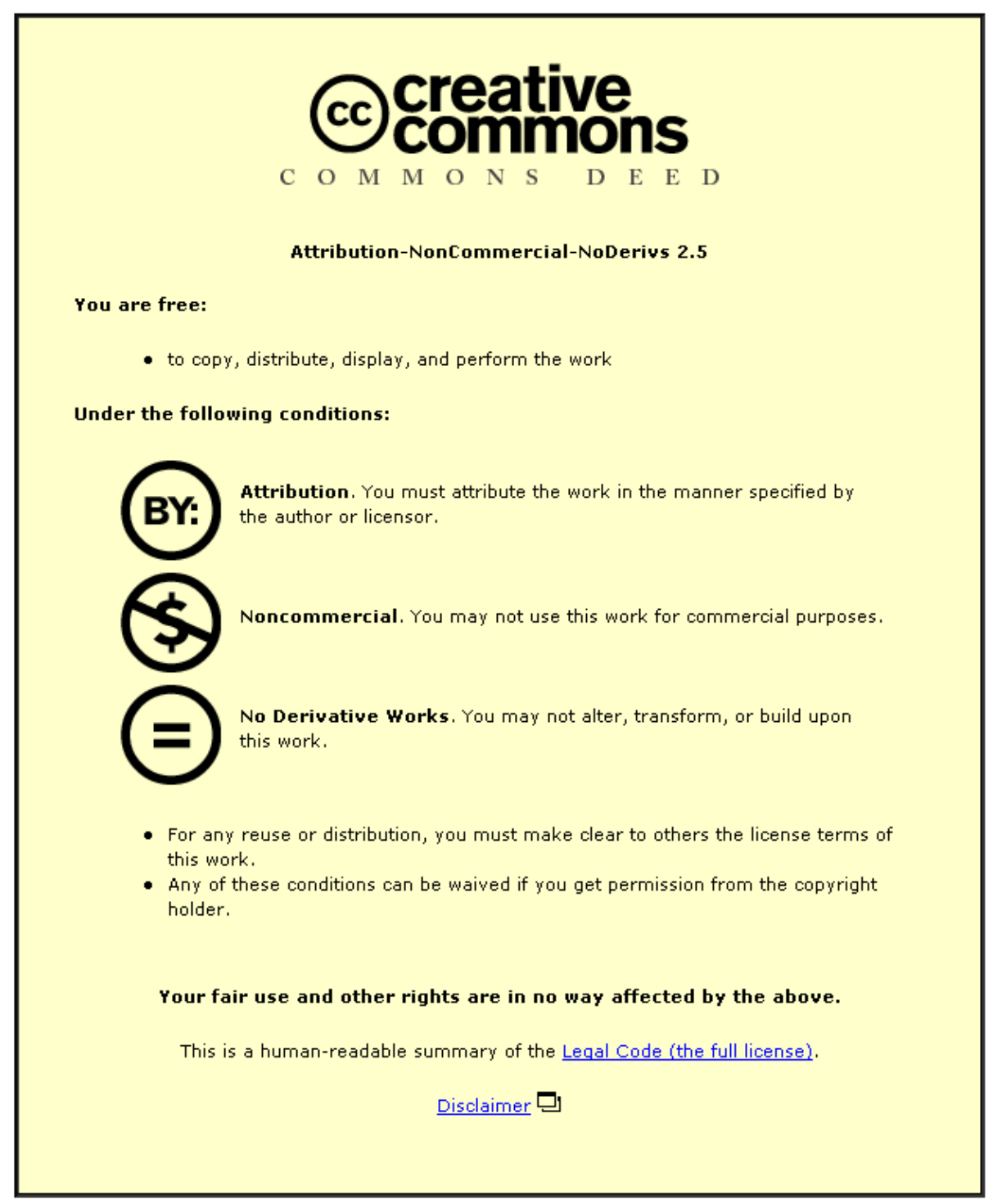

For the full text of this licence, please go to: http://creativecommons.org/licenses/by-nc-nd/2.5/ 


\title{
Condition Monitoring of Rail Vehicle Bogies
}

\author{
Christopher P. Ward* Roger M. Goodall* Roger Dixon* \\ Guy Charles** \\ * Department of Electronic and Electrical Engineering, Loughborough \\ University, Loughborough, Leicestershire, LE11 3TU, UK(e-mail: \\ c.p.ward@lboro.ac.uk; r.m.goodall@lboro.ac.uk; r.dixon@lboro.ac.uk) \\ ** School of Engineering, The University of Nottingham, University \\ Park, Nottingham, NGr 2RD, UK(e-mail: \\ Guy.Charles@nottingham.ac.uk)
}

\begin{abstract}
This paper details a range of work carried out by the authors within the general theme of advanced condition monitoring possibilities for rail vehicle bogies. Maintenance of rail vehicle bogies represents one of the largest areas of whole vehicle running costs and their efficient operation is of safety critical importance to the entire rail system. Currently the majority of maintenance is carried out on a scheduled basis which can be time consuming, costly and potentially not effective at fault detection. This paper reviews concepts that could allow real time detection of the condition of the bogie so as to reduce vehicle out of service time and improve safe operation. Concepts reviewed are: the use of condition monitoring for detection of suspension component condition; detection of low adhesion conditions; and assessment of the wheel-rail interface condition.
\end{abstract}

Keywords: Accelerometers, Fault Diagnosis/Detection, Kalman Filters, Nonlinear Systems, Piecewise Linear Analysis, Recursive Least Squares, Railways, Vehicle Dynamics

\section{INTRODUCTION}

This paper details a range of work carried out by the authors for a project within the Rail Research UK collaboration, the general theme of which was condition monitoring around the rail vehicle bogie system.

Maintenance of the rail vehicle bogie represents one of the largest areas of whole vehicle running costs, and its efficient operation is of critical importance to the safety of the entire rail system, Iwnicki (2006). The bogie system must provide the guidance for the rail vehicle, its running stability, isolate the passengers or cargo from disturbances at the rail and possibly provide the tractive effort for the vehicle, Wickens (2003).

Currently, most bogie system maintenance is performed on a scheduled basis, when critical components are checked for functionality. Key amongst these are suspension components like the secondary dampers; adhesion characteristics of the wheel-rail contact; and the geometric shape of the wheel-rail contact. All of these components can change their parameters rapidly in operation and as such can present safety issues if they alter outside of an inspection period and are not detected.

The key concept presented here is the use of condition monitoring to give a real time estimate of the parameters of these components. Ideas for monitoring have been employed in simulation in conjunction with data gathered from a real system. These techniques include; the use of particle filters for estimation of damper coefficients, Li et al. (2006), Kalman filters estimation of real time adhesion characteristics of the wheel-rail interface, Charles et al. (2008c) and various Kalman filter and system identification techniques for the estimation of the wheel-rail contact geometry, Charles et al. (2008b) and Charles et al. (2008a). More specifics of contemporary work on the latter are covered briefly in Ward et al. (2010b) and more thoroughly in Ward et al. (2010a).

These techniques present the potential to progress from scheduled calendar based maintenance to unscheduled condition based maintenance, cutting operational costs. Demand for these estimation techniques is generated by systems such as the operational Bombardier ORBITA, Bombardier (2010), where maintenance requirements are generated automatically through on train sensors, then dispatched to the relevant maintenance depot; and the European Train Control System, UIC (2010), in which a rail vehicle will be expected to brake at any position in the network where adhesion conditions may not be known, consequently making real time estimation of this important parameter a necessity.

\section{THE RAILWAY BOGIE}

The railway bogie is a complex, multi-degree of freedom system that is tasked with multiple roles in an extremely harsh environment, Figure 1 shows an example bogie. They consist of a bogie frame and two wheelsets of two coned wheels solidly fixed to a central shaft. The wheelsets run in a bearing house referred to as the axlebox, loads from which are transmitted to and from the bogie frame via the primary suspension. The vehicle body is then 


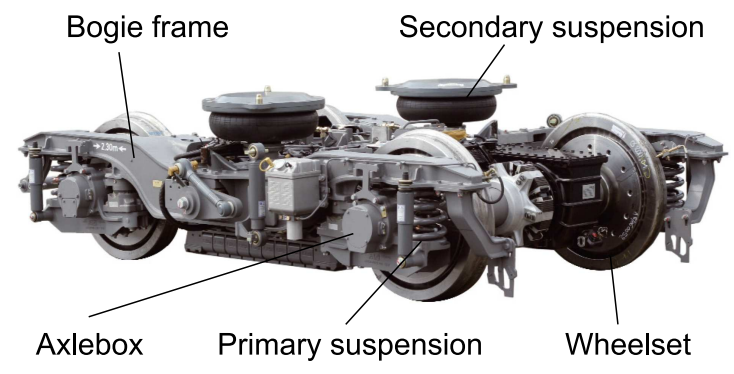

Fig. 1. Bombardier Transportation Commuter/Regional train FLEXX bogie

suspended by the secondary suspension from the bogie frame.

This system must principally provide guidance on both straight track and through curves, ensure dynamic stability and provide ride comfort to the passengers in the vehicle. Failure mode studies have shown that the majority of vehicle faults emanate from faulty wheel profiles and suspension components, Goodman et al. (2005), Weston et al. (2003).

Currently the majority of bogie systems are checked on a scheduled basis, with any condition monitoring in practice performed through signal processing and knowledge based assessments, Sunder et al. (2001). Therefore, potential exists to improve the performance of condition monitoring if a priori knowledge is used through a model of the system, Li and Goodall (2004).

\section{MODEL BASED CONDITION MONITORING}

The work carried out by the authors has been driven by a number of fundamental aims; the techniques employed generally use: a system model; advanced filtering; and relatively inexpensive inertial sensors. This last point means that the techniques can feasibly be applied to every bogie and wheelset in a train formation, meaning that the entire vehicle can be monitored in real time. The signals from the sensors also do not have to be used exclusively for condition monitoring of the bogie, they can also be used for track quality assessments, Weston et al. (2006) and vehicle speed estimation, Mei and Li (2008).

Therefore, this section presents work that follows these principles to estimate safety critical feature around the rail vehicle bogie. These are: suspension component parameters; wheel-rail adhesion forces; and three approaches to wheel-rail profile estimation.

\subsection{Suspension component parameter estimation}

Estimation of suspension parameters was originally reported in $\mathrm{Li}$ et al. (2006), Li et al. (2003) and $\mathrm{Li}$ et al. (2004). These sources described the use of a RaoBlackwellized Particle Filter (RBPF), Arulampalam et al. (2002), to determine the condition of secondary lateral dampers and secondary yaw dampers. Additionally the condition of the wheel-rail interface geometry was estimated through a single value conicity function, $\lambda$.

This work was first developed in simulation where the system was modelled on a Class 175 Coradia vehicle, but as a half vehicle system, with a single bogie and two wheelsets (Figure 2). The system was also only considered in the lateral and yaw direction of interest, due to minimal dynamic coupling to other planes.

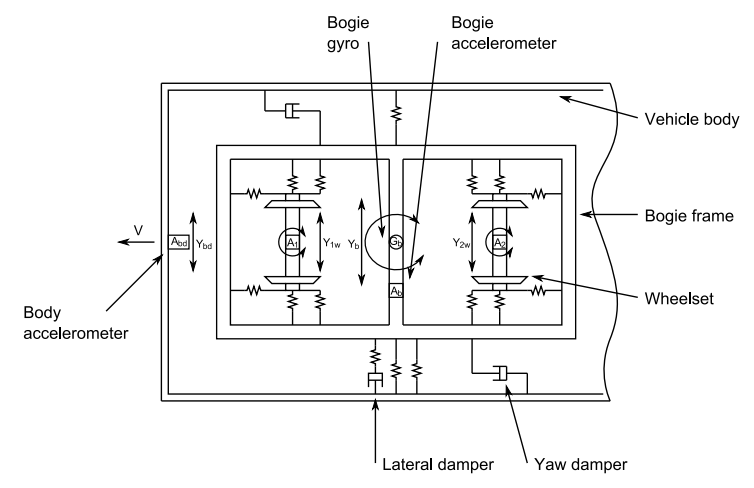

Fig. 2. Class 175 half vehicle model

Initial investigations in simulation utilised the full sensor set available on the wheelsets, the bogie and the body. A detailed statistical knowledge of the lateral track irregularity was also assumed. Under these assumptions the damping coefficient of the secondary lateral and yaw dampers, plus the conicity value could be estimated with some confidence.

The second investigation in simulation eliminated all of the sensors mounted on the wheelset. This is beneficial in application as mounting sensors in the harsh, unsprung environment of the axlebox can be costly and technically challenging. The estimation produced had some reduction in confidence of fit in comparison to the full sensor estimation, but was of sufficient quality for application. Robustness was tested by changing the statistical properties of the track alignment used for the estimation in comparison to that used in the model. The suspension parameters continued to be estimated with confidence, however the conicity parameter was widely divergent from that expected.

Finally, data was gathered from runs on the rail network, and the techniques were applied to the signals offline. Figure 3 shows the resultant estimations. Concurrent with simulation the estimation converges to the expected values for the suspension components, but due to a disparity of the track alignment statistics the conicity parameter fails to converge on the expected value.

Though results were mixed the application validated the use of model based condition monitoring for certain components around the rail-vehicle bogie system. It also highlighted the difficulty in estimating the wheel-rail interface geometry without a detailed knowledge of the track misalignment, hence this was further developed in section 3.3.

\subsection{Low adhesion estimation}

Low adhesion conditions at the wheel-rail interface remain a fundamental problem for many railways worldwide. They can lead to severe disruption of timetabled running due to conservative driving and in extremis can lead to signals being passed at danger. This is particularly prevalent during the leaf fall season when leaf film builds up on 

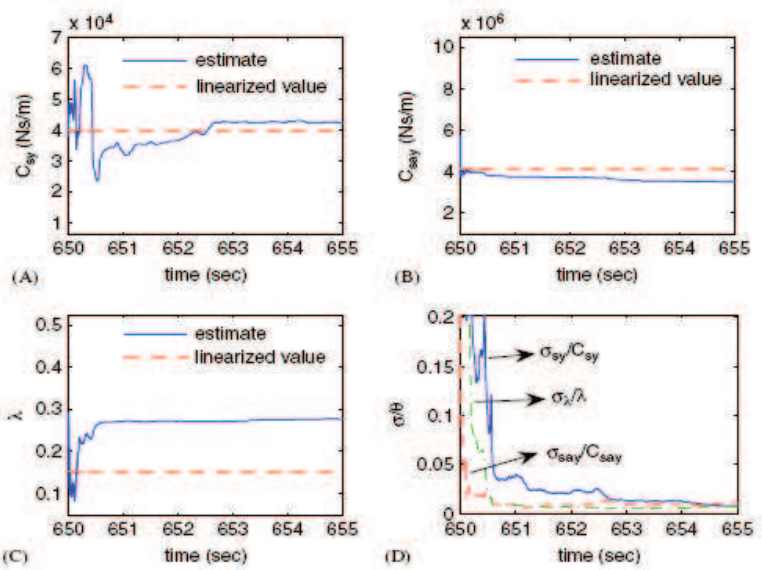

Fig. 3. Results of parameter estimation using data from real tests: (A) estimate of $C_{s y}$; (B) estimate of $C_{s a y}$; (C) estimate of $\lambda$; (D) ratio of the STD over parameter estimates

the rails causing adhesion coefficients to drop considerably, Cann (2006). Mitigation steps include sanding of the rails, grinding of the railhead, laser removal of contaminants, ceramic particle jetting and application of artificial friction material such as Sandite, Vasic et al. (2003). All of which are costly and applied in such a way that the benefits of their use can be difficult to assess. Also, they can not guarantee the friction level of the rail, hence a real time system that can identify local low adhesion conditions is highly desirable.

The work highlighted here was first shown in Charles et al. (2008c) and uses a model based Kalman filter estimation. The technique relies upon the knowledge that lateral forces generated for guidance are vectorised components of those used for braking performance. As with the previous estimation, the model used was a plan view, half body vehicle with a single bogie and two wheelsets, with ideal sensors. Forces at the wheel-rail contact point are calculated as a function of creep and can be linearised using Kalker coefficients, Kalker (1967). This assumption is normally sufficient for stability analysis and controller design purposes, but in this case it is important to understand the creep forces up to and beyond the saturation levels. Use was therefore made of the contact force model of Polach (2005), that is effectively a curve fitting mechanism.

Experimentation has shown that contrary to the expectation the initial slope of the creep curve varies with different adhesion properties, Pearce and Rose (1985), Harrison and McCanney (2002). Figure 4 shows this effect for varying conditions for a fixed contact patch size and load. This property enables different levels of adhesion to be detected during normal unsaturated running.

The estimation approach applied used a Kalman filter, Kalman (1960), to estimate the contact forces in the wheel-rail contact. This therefore ignores the complex nonlinear relationships and considers the system as a rigid body 'floating' on a series of contact patches. Initial estimation of the longitudinal forces proved unobservable, though proved successful when applied to the lateral and yaw forces. Simulations showed that the estimations

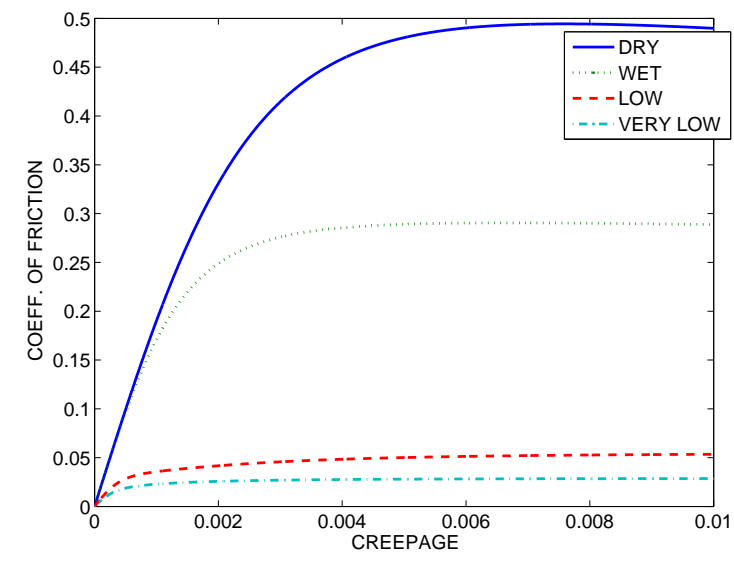

Fig. 4. Creep curves for dry, wet, low and very low adhesion conditions (wheel load of $4000 \mathrm{~kg}, 20 \mathrm{~m} / \mathrm{s}$ )

could not distinguish between the creep and gravitational forces so had to be estimated as one. This configuration showed some difference between the time data of the output from the model and estimation, however varying adhesion conditions can be detected by looking at the Power Spectral Density (PSD) of the estimated creep force and creep moment time samples. A reduction in the peak of the PSD can be observed as the adhesion values reduce, Figure 5.
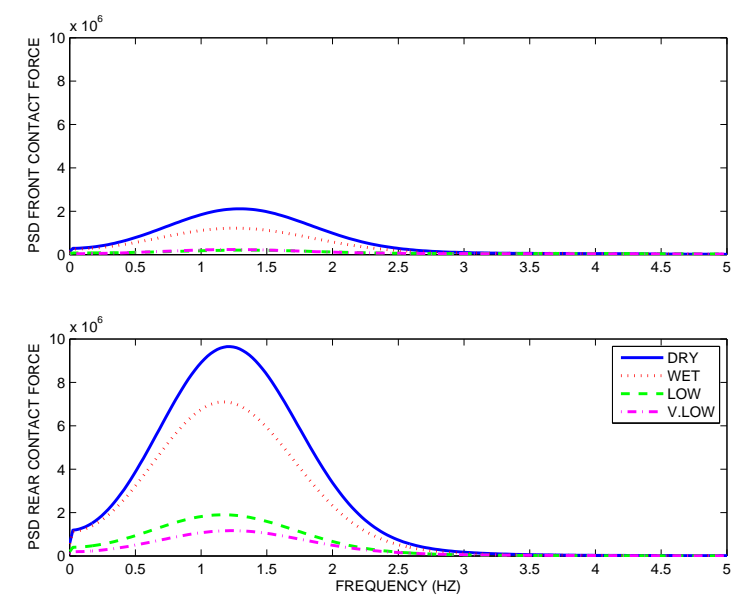

Fig. 5. PSD of the Kalman filter estimates of the lateral contact forces on the front and rear wheelsets for varying adhesion conditions

This work is currently the subject of further development with interest from the rail industry. This will look at how the force estimation will be used in practice and will be validated with data from multi-body dynamic simulations, roller rig tests and potentially full scale tests.

\subsection{Wheel-rail profile estimation}

As stated earlier the wheel-rail contact interaction is one of the most important elements of the rail system, the characteristics of which govern the rail vehicles' tangent track stability, cornering performance and ride quality. This relationship degrades with time as the tread of the wheelsets and the railhead wear with use. This is usually monitored separately and on a scheduled basis. The ideas 
shown here are for the real time estimation of the property with the rail vehicle in operation.

The model used for this speculative work is a simple single wheelset and suspended mass (Figure 6). Due to

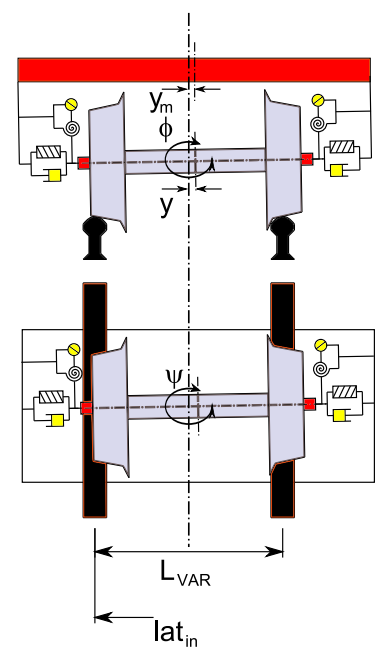

Fig. 6. Schematic of the single wheelset and single mass model used for the wheel-rail profile estimation

the low coupling across planes the wheelset dynamics are considered only in the lateral and yaw dimensions, with the suspended mass considered only in the lateral direction. Various models have been used for simulation and are taken from Garg and Dukkipati (1984), with exact model equations given in Ward et al. (2010a). The highest level models use linear Kalker approximations for the friction conditions, with static nonlinearities present as rolling radii and contact angles, $\left(r_{L}, r_{R}, \delta_{L}, \delta_{R}\right)$, that are functions of the relative wheel-rail displacement. These are calculated for specific combinations of wheel tread and railhead shape using a Newton-Raphson based iterative approach, Wickens (2003).

Simplification of this model can be made by linearising the system around the central portion of the running surface. This is the industrial norm for analysis and generates a function known as conicity, $\lambda$. The assumption is now that there is point contact between the wheel and the rail, and is represented by four relationships

$$
\begin{aligned}
& \frac{1}{2}\left(r_{L}-r_{R}\right)=\lambda y ; \frac{1}{2}\left(r_{L}+r_{R}\right)=r_{0} ; \\
& \frac{1}{2}\left(\delta_{L}-\delta_{R}\right)=0 ; \frac{1}{2}\left(\delta_{L}+\delta_{R}\right)=\lambda ;
\end{aligned}
$$

Further simplifications can be made by ignoring the lowered valued creep force terms, Wickens (2003). Again it is also assumed that ideal sensors are present for all of the relevant states and that the lateral irregularity of the track alignment is known for some of the estimations.

Three distinct methods for geometry estimation have so far been proposed, they are: nonlinear conicity estimation using Kalman filtering; nonlinear conicity estimation using system identification; and contact geometry estimation using piecewise linear system identification.

Conicity estimation through Kalman filtering The initial stages of the work used an Extended Kalman filter to es- timate a generic nonlinear smooth continuous conicity parameter applied to the linearised equations, Charles et al. (2008c). Initially the state vector was augmented with just the conicity parameter, but this failed to converge. The state vector was therefore additionally augmented to estimate the unknown track disturbance, $d$. This estimation in simulation is acceptable, but uncertainty increases at lower conicity values.

The estimation can be improved further by adding extra dynamic understanding about how the conicity function varies with the lateral displacement in the form $\dot{\lambda}=\frac{d \lambda}{d y} \dot{y}$ and $\dot{d}=0$. This process now stores a lookup table of corresponding relative wheel-rail displacements that is used to give the Kalman filter some insight into the variability of the conicity. The results from the process are shown in Figure 7, and show that around small displacements there is an excellent fit, but at larger displacements where there may be a lack of data due to running conditions the fit fails.

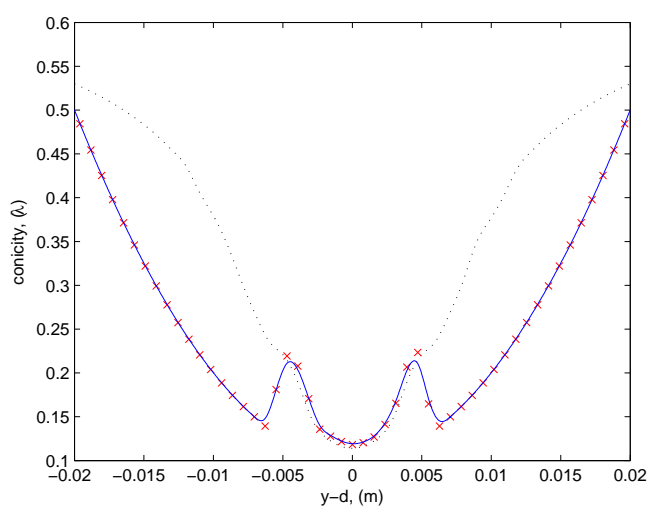

Fig. 7. Kalman filter updating estimation of the conicity function

Conicity estimation through system identification Although Kalman filtering can be used successfully as a parameter estimator an alternative approach is to use system identification, where the Kalman filter was also used to estimate the input signal to the system. System identification requires a detailed knowledge of the track alignment, that is possibly very difficult to measure in real time. This work was first shown in Charles et al. (2008b) and Charles et al. (2008a).

System identification basics are covered in numerous sources, Söderström and Stoica (1994), Ljung (1999), Aström (1989), but essentially involve fitting parameter values to a set of measured regressors or states to minimise the square of the error of the estimated output to the real output. This can be performed in a 'black box' manner where there is no a priori knowledge of the internal structure of the system, or in a 'grey box' manner where there is some knowledge of the structure and the identification is used to find the correct parameters for the unknown states or regressors. The parameters are not strictly restricted to being linear, but can be high order terms, Seber and Wild (2003), or multiple Piecewise Cubic Polynomials (PCP), Ichida et al. (1976). 
A combination of the PCP and 'grey box' approaches were used here and applied to the simplified version of the linearised equations, with the results shown in Figure 8. This produces a very close fit to the nonlinear shape of

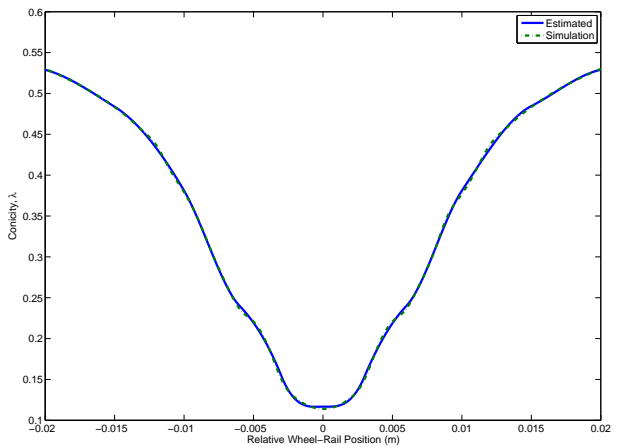

Fig. 8. Least squares estimated conicity function with PCP function

the conicity function, however it should be noted that in practice the conicity may be discontinuous and therefore less well matched to the smoothing PCP function.

Contact geometry estimation by system identification When the identification procedure of the previous section was applied to the more advance system model with nonlinear contact geometries the conicity estimates were of poor quality due to the estimation model being insufficient to fit to the complex dynamics of the simulation model. An alternative approach was sought to estimate the contact angles and rolling radii directly, this was first briefly covered in Ward et al. (2010b) and in more detail in Ward et al. (2010a).

The nonlinearities in the model present themselves as four geometric combinations, $\left(r_{L}+r_{R}\right),\left(r_{L}-r_{R}\right),\left(\delta_{L}+\delta_{R}\right)$, $\left(\delta_{L}-\delta_{R}\right)$. Using real railhead $(113 \mathrm{~A})$ and wheel $(\mathrm{P} 8)$ profiles, these are now complex nonlinear discontinuities. This geometric nature makes it difficult to fit a single function across the entire range of displacement, therefore it was decided to perform a series of identifications across the range of relative displacement to build up a table of values.

The models were also further developed to include the effects of gauge width variation, that can significantly affect the geometric relationships. Also the system was excited by measured geometry variation from a track recording car, as using filtered noise sources can cause frequency biasing that can lead to misleading results, Evans and Berg (2009).

The estimation method first involves ordering the data in relation to the wheel-rail displacement, then performing 'grey box' system identifications in a recursive manner so that the estimates can be updated as new data is collected in real time. Initial estimates were performed with no gauge width variation present, and as expected this gives a near perfect estimation. The second estimation was performed with gauge width variation present, this adds a large degree of uncertainty to the estimation and as such the estimation occupies a 'space' rather than being a single value function, Figure 9. Though not shown, the

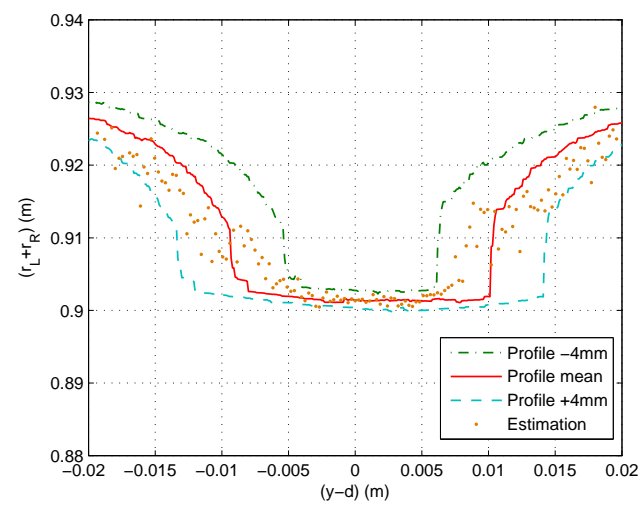

Fig. 9. Recursive least squares estimate of the rolling radii sums with gauge width variation

estimation for $\left(\delta_{L}+\delta_{R}\right)$ fails to converge as this may be dependent upon the gauge width being precisely known.

\section{FUTURE CHALLENGES}

Presented in the previous sections are potential algorithms for real time estimation of key parameters around the rail vehicle bogie. These have so far been software based demonstrations and not applied on a real system. Therefore the key challenge is to ensure that the algorithms work on a multi-bodied dynamic simulation, and that they work in an efficient manner so can be applied to processors present on railways vehicles that may be many times less powerful than a standard desktop computer.

The simulations all assumed an ideal placement of the sensors on the vehicle, the bogie and the axlebox. This may not be physically possible on many vehicles, as such a sensor placement exercise will need to be undertaken to trade-off the key compromises of position, cost and signal to noise ratio. Also, their placement will need to be compatible with the conflicting demands of other detection algorithms that will also use the data generated.

\section{CONCLUSION}

This paper reviewed work that was set in the general context of developing real time condition monitoring algorithms for safety critical components around the rail vehicle bogie system. These techniques will potentially help reduce the out of service time of rolling stock due to maintenance and help with the safe running of the network.

Three key techniques highlighted were: suspension damper monitoring using RBPF, which was proven using data collected from the network; a low adhesion estimation concept that was shown to detect varying conditions in simulation; and various methods of wheel-rail profile estimation.

Discussion was also made of the need to develop these algorithms so that they can be applied efficiently in realtime and the challenges of sensors placements that balance the needs of various condition monitoring algorithms that will use their signals. 


\section{ACKNOWLEDGEMENTS}

The authors would like to thank Rail Research United Kingdom (RRUK) and the Engineering and Physical Science Research Council (EPSRC) who funded this research. Additional thanks go to the Rail Technology Unit (RTU) at Manchester Metropolitan University (MMU) who provided track and vehicle data for a section of the study.

\section{REFERENCES}

Arulampalam, M., Maskell, S., Gordon, N., and Clapp, T. (2002). A tutorial on particle filters for online nonlinear/non-gaussian bayesian tracking. IEEE Transactions on Signal Processing, 50(2), 174-188.

Aström, K. (1989). Adaptive control. Addison-Wesley, first edition.

Bombardier (2010). Orbita - predictive asset management, the future of fleet maintenance. http://www.bombardier.com/en/transportation/ accessed 7th April 2010.

Cann, P. (2006). The "leaves on the line"' problem - a study of leaf residue film formation and lubricity under laboratory test conditions. Tribology Letters, 24(2), 151158.

Charles, G., Dixon, R., and Goodall, R. (2008a). Condition Monitoring Approaches to Estimating Wheel-Rail Profile. In Proceedings of UKACC Control Conference, Manchester.

Charles, G., Goodall, R., and Dixon, R. (2008b). A Least Mean Squared Approach to Wheel-Rail Profile Estimation. In Proceedings of the 4th International Conference On Railway Condition Monitoring.

Charles, G., Goodall, R., and Dixon, R. (2008c). Modelbased condition monitoring at the wheel-rail interface. Vehicle System Dynamics, 46(1), 415-430.

Evans, J. and Berg, M. (2009). Challenges in simulation of rail vehicle dynamics. International Journal of Vehicle Mechanics and Mobility, (8), 1023-1048.

Garg, V. and Dukkipati, R. (1984). Dynamics of Railway Vehicle Systems. Academic Press, first edition.

Goodman, C., Ling, C., Li, P., Weston, P., Goodall, R., and Roberts, C. (2005). Condition monitoring of railway track and vehicle suspension using an in-service train. In Proceedings of the IEEE conference on railway engineering, Hong Kong and Shenzen, China.

Harrison, H. and McCanney, T. (2002). Recent developments in coefficient of friction measurements at the rail/wheel interface. Wear, 253(1), 114-123.

Ichida, K., Yoshimoto, F., and Kiyono, T. (1976). Curve Fitting by a Piecewise Cubic Polynomial. Computing, 16(1), 329-338.

Iwnicki, S. (2006). Handbook of Railway Vehicle Dynamics. Taylor and Francis, first edition.

Kalker, J. (1967). On the Rolling Contact of Two Elastic Bodies in the Presence of Dry Friction. Ph.D. thesis, Delft University of Technology, Delft, Netherlands.

Kalman, R. (1960). A new approach to linear filtering and prediction. Transactions of ASME - Journal of Basic Engineering, 35-45.

Li, P. and Goodall, R. (2004). Model-based condition monitoring for railway vehicle systems. In Proceedings of the UKACC international conference on control, Bath, $U K$.
Li, P., Goodall, R., and Kadirkamanathan, C. (2003). Parameter estimation of railway vehicle dynamic model using rao-blackwellised particle filter. In Proceedings of the seventh European control conference, Cambridge, $U K$.

Li, P., Goodall, R., and Kadirkamanathan, C. (2004). Estimation of parameters in a linear state space model using a rao-blackwellised particle filter. IEE ProceedingsControl Theory and Applications, 151:727-738.

Li, P., Goodall, R., Weston, P., Ling, C., Goodman, C., and Roberts, C. (2006). Estimation of railway vehicle suspension parameters for condition monitoring. Control Engineering Practice, 15:43-55.

Ljung, A. (1999). System Identification, Theory for the User. Prentice Hall, second edition.

Mei, T. and Li, H. (2008). Measurement of vehicle ground speed using bogie based inertial sensors. IMechE proceedings, Part F: Rail and Rapid Transit, 222(2), $107-116$.

Pearce, T. and Rose, K. (1985). Measured force-creep relationships and their use in vehicle response calculations. In Proceedings of the IAVSD 9th Symposim, Linkoping.

Polach, O. (2005). Creep forces in simulations of traction vehicles running on adhesion limit. Wear, 258(1), 9921000 .

Seber, G. and Wild, C. (2003). Nonlinear Regression. Wiley, first edition.

Söderström, T. and Stoica, P. (1994). System Identification. Prentice Hall, second edition.

Sunder, R., Kolbasseff, A., Rohm, A., and J., W. (2001). Operational experiences with onboard diagnosis for high speed trains. In Proceedings of the World Congress on Railway Research, WCRR2001.

UIC (2010). ERTMS. http://www.uic.org/ accessed 7th April 2010.

Vasic, G., Franklin, F., and Kapoor, A. (2003). New rail materials and coatings. Technical Report RRUK/A2/1, University of Sheffield, prepared for RSSB. http://portal.railresearch.org.uk/RRUK/Shared \%20Documents/rssba2a.pdf.

Ward, C., Goodall, R., and Dixon, R. (2010a). Wheelrail profile condition monitoring. In Proceedings of the UKACC control conference, Coventry.

Ward, C., Weston, P., Stewart, E., Li, H., Goodall, R., Roberts, C., Mei, T., Charles, G., and Dixon, R. (2010b). Condition monitoring opportunities using vehicle based sensors. Awaiting publication: IMechE proceedings , PartF: Rail and Rapid Transit.

Weston, P., Roberts, C., Goodman, C., Goodall, R., Li, P., and Ling, C. (2003). Enhanced rail contribution by increased reliability (ercir) - instrumenting in-service rail vehicle to monitor vehicle and track. In Proceedings of the World Congress on Railway Research, WCRR2003, Edinburgh, Scotland.

Weston, P., Ling, C., Goodman, C., Roberts, C., Li, P., and Goodall, R. (2006). Monitoring lateral track irregularity from in-service railway vehicles. IMechE proceedings, Part F: Rail and Rapid Transit, 221(RRUK Special Issue), 89-100.

Wickens, A. (2003). Fundamentals of Rail Vehicle Dynamics: Guidance and Stability. Swets and Zeitlinger, first edition. 